\title{
Recherches et développement économique au Sénégal expériences et explorations
}

Daniel Thiéba

\section{(2) OpenEdition}

1 Journals

Édition électronique

URL : http://journals.openedition.org/apad/307

DOI : 10.4000/apad.307

ISSN : 1950-6929

Éditeur

LIT Verlag

Édition imprimée

Date de publication : 15 mars 1991

\section{Référence électronique}

Daniel Thiéba, « Recherches et développement économique au Sénégal expériences et explorations », Bulletin de l'APAD [En ligne], 1 | 1991, mis en ligne le 23 juin 2006, consulté le 08 septembre 2020. URL: http://journals.openedition.org/apad/307 ; DOI : https://doi.org/10.4000/apad.307

Ce document a été généré automatiquement le 8 septembre 2020

Bulletin de l'APAD 


\title{
Recherches et développement économique au Sénégal expériences et explorations
}

\author{
Daniel Thiéba
}

Quelques expériences anciennes

1 Les recherches en sciences sociales et les efforts et initiatives menés pour articuler recherche et développement ont eu pour cadre principal l'ISRA, pour le Sénégal. Aussi, c'est à partir de l'expérience de cette institution que nous fonderons notre présentation. Il s'agit donc d'une présentation des variations des théories et méthodologies du développement économique, particulièrement dans le secteur agraire.

Les recherches en sciences sociales impulsées se sont effectuées dans le Département "Systèmes de Production" de l'ISRA. Les recherches en anthropologie se sont fondées sur les structures, rapports de production, de pouvoir et les intérêts économiques des acteurs sociaux, conformément au paradigme wébérien: le phénomène $M$ est une fonction des actions $\mathrm{m}$, lesquelles dépendent de la situation $\mathrm{S}$ de l'acteur, cette situation étant elle-même affectée par des données macrosociales M (Raymond Boudon, La place du désordre, PUF 1984, p.40). Les propositions se sont voulues empiriques-contextuelles, évitant une généralisation dans le sens d'une Théorie (prudence heureuse). Les connaissances accumulées ont permis de mieux comprendre les logiques et stratégies sociales des producteurs.

Dans le cadre d'équipes pluridisciplinaires, les analyses anthropologiques étaient plus ou moins prises en compte. Lorsqu'elles ne l'étaient pas, on continuait d'affubler les producteurs de mentalité rétrograde.

Dans la seconde éventualité, les chercheurs agronomes et zootechniciens étaient invités à l'élaboration de nouvelles propositions. En tenant à prendre en compte les travaux anthropologiques, les chercheurs des autres disciplines et les développeurs les plus en pointe se sont enfermés dans un processus de bricolage qui ne parvenait que rarement à concilier leur logique et celle des producteurs. 
A la recherche de procédures participatives

De nouvelles démarches, impulsées par des équipes promouvant l'approche systématique, ont mis en œuvre une approche se fondant sur la participation d'associations de producteurs. Cela signifie que ceux-ci sont associés en tant que groupe aux procédures suivantes: définition des priorités et des technologies souhaitables ; test dans les conditions réelles ; vulgarisation ; évaluation.

Des expériences sont en cours en Casamance dans le cadre d'une collaboration entre l'ISRA, l'ENEA (École Nationale d'Économie Appliquée) et le CADEF (association paysanne). Dans la région de Fatick, une collaboration similaire s'est instaurée entre l'ISRA et l'ARAF (Association Régionale des Agriculteurs de Fatick).

7 Cette démarche participative permet aux chercheurs, notamment ceux en sciences sociales de recueillir des résultats plus rapidement que par le moyen des approches traditionnelles; elle permet en outre d'impliquer plus profondément des producteurs dans l'essai en réelle grandeur et dans la vulgarisation. Il serait prématuré de faire un bilan de ce type d'approche, même si certains résultats sont observables. Il bénéficie présentement de l'appui de nombreuses institutions de financement qui poussent à sa généralisation par les chercheurs de toutes disciplines. Ce courant met à profit l'apport méthodologique anglo-saxon (Rapid Rural Appraisal: RRA). Il suscite un fort intérêt auprès des chercheurs africains.

On peut formuler une critique générale des paradigmes qui ont guidé les recherches pour le développement jusqu'à ce jour. On continue à raisonner comme si les logiques sociales étaient des données indépendantes dont il fallait tenir compte d'emblée, avant d'envisager la définition d'itinéraires techniques possibles.

Explorations

Dans le cadre d'un programme d'appui à une fédération de cinq groupements villageois, une collaboration est en cours entre l'INODEP (Institut Ecuménique pour le Développement des Peuples) et l'ISRA/Kaolack. Ce qui est visé, c'est la modification de la situation $\mathrm{S}$ et des données macro-sociales $\mathrm{M}$ par le biais d'une série d'actions menées par les organisations dont se sont dotées les acteurs sociaux. La problématique consiste ici à viser des réformes sociales, à élaborer de nouveaux construits sociaux afin de créer un environnement plus favorable aux logiques allant dans le sens du «développement». C'est un défi pour l'anthropologie qui se voit interpellée par de nouveaux champs: les organisations, l'action collective. Tâche ardue mais qui s'avère légitime. Celle-ci se tonde sur l'existence d'un tissu associatif qui se densifie tout en exprimant une demande d'appui. Nombre de ces associations sont fragiles, mais certaines d'entre elles recèlent des potentialités méconnues.

Il s'agit d'une recherche-action qui a pour préoccupation autant la production de connaissances que le changement et la capacité des acteurs sociaux "de se reconnaître et s'affirmer comme producteur plus que comme consommateur de la situation sociale, comme capable de mettre celle-ci en question au lieu de seulement lui répondre" (A. Touraine, "le retour de l'acteur", Fayard,1984 p. 38).

11 Cette approche rompt avec l'idée d'une recherche considérée comme un travail effectué par un chercheur sur un objet extérieur à lui. Le chercheur "produit son analyse par la mise sur pied d'un dispositif de collaboration entre le groupe ou l'organisation sociale en analyse et lui-même." (Rémi Hess, "La sociologie d'intervention" P.U.F, 1981). Il s'agit d'une sociologie d'intervention fondée sur les 
demandes nouvelles qui restent évidemment à clarifier, à reformuler. Dans cette présentation, la sociologie refuse de s'enfermer dans l'analyse du système social présent. Elle énonce la thèse suivante: s'il existe de nouvelles organisations, de nouveaux acteurs qui se donnent pour objectif explicite le changement social et le développement, il est légitime d'envisager des innovations sociales rendant plus faciles les innovations technologiques.

Bien que les recherches aient démarré, les objectifs essentiels restent à l'état de projet ; les enjeux et difficultés se précisent. Les demandes de ce type ne sauraient se constituer sans connaissance de ce que peut apporter la recherche, sans existence d'institutions à même de mettre en œuvre des procédures de recherche-action. L'intérêt de cette approche est qu'elle permet pour certains champs de la recherche l'obtention d'une connaissance difficilement accessible par les méthodes traditionnelles.

Quelles institutions pour la recherche-développement?

Pour conduire des recherches de ce type, il faut des institutions disposant d'une autonomie administrative et financière. Elles doivent avoir la souplesse requise pour traiter avec des partenaires multiples. Par excellence, elle est le lieu où des chercheurs interviennent de deux façons :

- en accordant une partie de leur temps pendant une durée relativement longue,

- en accordant la totalité de leur temps sur une courte période.

Ces modalités, définies en accord avec les organismes de recherches, leur donnent ainsi la possibilité de poursuivre des recherches fondamentales dans le cadre de l'Université ou d'institutions de recherches.

Il s'est constitué depuis quelques mois à Dakar l'antenne d'une institution (IRED : Innovations et réseau pour le développement) ayant le statut d'ONG internationale, avec pour vocation de répondre aux demandes d'associations paysannes sous la forme de recherche-action. Les pays suivants seront concernés: Sénégal, Mali, Gambie, Guinée-Conakry, Guinée-Bissau. Le financement est accordé par la Fondation Ford et le CRDI : (Centre de Recherche pour le Développement International, Canada). Ce type d'institution correspond -il aux conditions d'une relation féconde entre recherche et développement ? Il serait prématuré de répondre à une telle interrogation.

Dans le contexte actuel du Sénégal et plus généralement de l'Afrique, la sociologie/ anthropologie a conduit un grand nombre d'investigations de qualité qui demandent une réflexion synthétique en vue de l'élaboration d'un corps de théories du changement social à validité contextuelle. De nouveaux champs de recherches restent en friche (organisations, modes d'actions collectives...). Le grand défi est non pas "affinement de la production scientifique" en soi, mais probablement dans son articulation avec les demandes des organisations de base et des institutions d'appui au développement. 